\title{
Acute Lymphocytic Leukemia With KMT2A- ELL Fusion Mutation in a Patient With Untreated Chronic Lymphocytic Leukemia: A Case Report and Literature Review
}

\section{Shilin Zhang}

The First Hospital of Jinlin University https://orcid.org/0000-0002-5872-3418

\section{Sennan Qiao}

Institute of Frontier Medical Science of Jilin University

\section{Wei Han}

The First Hospital of Jilin University

\section{Ruiping $\mathrm{Hu}$}

The First Hospital of Jilin University

\section{Zhonghua Du}

The First Hospital of Jilin University

\section{Jing Bai}

Jilin University First Hospital

\section{Fuming Zhang}

The First Hospital of Jilin University

Sujun Gao ( $\nabla$ sjgao@jlu.edu.cn )

Jilin University First Hospital

\section{Case Report}

Keywords: acute myeloid leukemia, chronic lymphocytic leukemia, concurrent, KMT2A-ELL

Posted Date: March 9th, 2021

DOl: https://doi.org/10.21203/rs.3.rs-290097/v1

License: @ (i) This work is licensed under a Creative Commons Attribution 4.0 International License. Read Full License 


\section{Abstract}

Background: The concurrent of chronic lymphocytic leukemia (CLL) and acute myeloid leukemia (AML) in a patient is a rare situation, and has caused obstacles in clinical management.

Case presentation: In the current study, we described a 64-year old female who was characterized by intermittent fatigue, edema of both lower limbs, dyspnea, and occasionally fever up to $39^{\circ} \mathrm{C}$. The admission blood routine detections and the flow cytometry showed the patient was impaired by both CLL and AML. In RT-qPCR molecular detection, KMT2A-ELL fusion gene t (11:10) (q23:p13.1) was detected, which was verified by FISH detections. The next-generation sequencing (NGS) revealed a missense mutation of p.V157F in the coding sequence of TP53 gene, and frameshift mutations of p.V220fs and p.A382fs in the coding sequence of WT1.

Conclusions: Collectively, the patient reported in this case was simultaneously impaired by CLL and AML. Our findings also inferred that the concurrent of CLL and AML might be attributed to the fusion mutation in KMT2A-ELL gene.

\section{Introduction}

Chronic lymphocytic leukemia (CLL) is a mature B cell neoplasm that induces immunosuppression state in patients and increases the incidence to develop other cancer types. Based on previous investigations, CLL patients have a much higher risk for developing malignancies such as lung cancer, melanoma, etc., compared with general population (1). Worse still, in addition to develop solid tumors, CLL patients are associated with a 10 -fold higher incidence of being impaired by subsequent multiple myeloma $(2,3)$ such as myelodysplastic syndrome (MDS) and acute myeloid leukemia (AML) (4). However, only tens of CLL concurrent with other hematologic malignancies have been described since 1970s (5-7). Currently, the development of subsequent multiple myeloma in patients with untreated CLL is generally diagnosed as therapy-related, and the prognosis of those patients is always inferior to that of de novo cases (8). Thus, the comprehensive reveal of diagnosis and clinical features of CLL patients concurrent with other hematologic malignancies is critical for the improvement of their survival rate. In the current study, we report a CLL patient who developed rare KMT2A-ELL-fusion AML and review the literature to demonstrate the clinical characteristics of such patients.

\section{Case Presentation}

\section{Case history}

A 64-year-old female presenting a six-hour history of waist abdomen ache associated with intermittent vomiting was admitted by our hospital at 2nd Feb 2020. The patient had an eight-year history of diabetes mellitus and a seven-year history of coronary heart disease (CHD). She denied all other medical histories, including hypertension, transfusion, hepatitis, tuberculosis, smoking, drinking and allergies. The 
admission examination showed that the patient was 165-cm height and 71-kg weight with clear conscious, autonomous position and anemic appearance. She was also characterized by anemic appearance and had normal skin elasticity, and no swelling of superficial lymph nodes was detected. She was impaired by lower abdominal pain without rebound tenderness, muscle tightness, abdominal masses, and Murphy sign, but with left kidney area percussion pain (+). During the last two months, the patient reported intermittent fatigue, edema of both lower limbs, dyspnea, and occasionally fever up to $39^{\circ} \mathrm{C}$. The detection results of CT showed that the patient had infections in bronchitis and both lungs, multiple lymph nodes in the abdominal cavity and retroperitoneum, obstruction of the left ureter, and fatty liver. The detection results of urine routine showed that she also had urinary system infections. The detection results of blood routine examination revealed followings: the level WBC was $15.18 \times 10^{9} / \mathrm{L}$ with 0.03 neutrophils (absolute value $0.54 \times 10^{9} / \mathrm{L}$ ), the level of lymphocytes was 0.84 (absolute value $13.37 \times 10^{9} / \mathrm{L}$ ), the level of monocytes was 0.12 (absolute value $1.93 \times 10^{9} / \mathrm{L}$ ), the level of nuclear cells was $1.93 \times 10^{9} / \mathrm{L}$, the level of red blood cells was $1.93 \times 10^{12} / \mathrm{L}$, the level of hemoglobin was $70 \mathrm{~g} / \mathrm{L}$, and the level of hematocrit was $0.214 \mathrm{~L} / \mathrm{L}$. The mean corpuscular volume (MCV) was $110.9 \mathrm{fL}$ with corpuscular hemoglobin of $36.3 \mathrm{pg}$, ferritin of $697.0 \mathrm{ug} / \mathrm{L}$, reticulocytes $4.11 \%$, and platelets of $93 \times 10^{9} / \mathrm{L}$.

\section{Materials And Methods}

The patient was then transferred to the Department of Hematology of the Cancer Center after multidisciplinary consultation and subjected to detections of bone marrow morphology, bone marrow biopsy, bone marrow immunochemistry, karyotype analysis, flow cytometry, RT-qPCR, and high throughput sequencing for explicit diagnosis. All the procedures of the current study were performed in accordance with the Declaration of Helsinki and were approved by the ethic committee of The First Hospital of Jilin University. The patient had signed a written informed consent form for related screening, inspection, and data collection before surgeries.

\section{Results}

The detection of bone marrow morphology with Wright staining showed active hyperplasia of nucleated cell. Moreover, the proportion of granule cells of was significantly reduced $(0.5 \%)$ and erythroid cells $(1 \%)$ had normal morphology. Mature red blood cells were slightly different in size, and the proportion (33\%) of monocytes increased with uneven distribution. Primitive naive monocytes accounted for $23 \%$, lymphocytes accounted for $67.5 \%$, and immature lymphocytes accounted for $3.5 \%$ of the total proportion. Only 16 megakaryocytes were observed in the smears of blood with platelets distributed in piles. The smears also showed an increase in lymphocytes with primitive monocytes accounting for $3 \%$ of the total cell proportion (Fig. 1A).

HE staining showed that the proportion of bone marrow nucleated cells was regular, while the proportion of myeloid blast cells increased (accounting for 50-60\%) with diffusive distribution (Fig. 1B). The mature granular and red cells were scattered distributed barely observed in the samples. Few megakaryocytes 
were observed. A few lymphocytes were distributed in clusters, and reticular fiber staining showed MF2 grade, focal. It was also shown that bone marrow samples were $100 \%$ negative for pathological cells, $22 \%$ positive for specific esterase ASD (score 22), negative for non-specific esterase a-NAE, extrairon ++ for iron staining (Fig. 1C). The results of immunohistochemistry detection showed mature lymphocytes were positive (stained brown) for CD34, CD117, MPO, while negative for CD3, CD20, and CD5 (Fig. 1D and Fig. 1E).

The analysis of karyotype identified nine mitosis phases, all of which were normal karyotype: $46, X X[9]$.

The flow cytometry showed that T cell occupies $14.22 \%$ of nuclear cells (Fig. 2), positive in the detections of CD34, CD117dim, CD123, CD43, CD13, CD38, CD33, and HLA-DR, partially positive in the detections of CD19dim and CD200dim, and negative in the detections of CD5, CD7, CD2, CD10, CD20, CD138, CD81, CD23, CD11c, FMC7, CD103, CD22, CD25, CD36, CD11b, CD16, CD14, CD64, CD15, CXCR4, CD79b, cCD3, cmpo, cCD79a, mKappa, which were identified as abnormal myeloid protocells (Fig. 2). CD19 + cells accounted for $58.1 \%$ of the total nuclear cells, positive in the detections of CD19dim, CD5dim, CD81 dim, CD200, CD79b, CD43, CD23, CD22dim, HLA-DR, mKappadim, partially positive in the detections of CD20dim, CXCR4, and negative in the detections of CD34, CD117, CD38, CD7, CD2, CD10, CD138, FMC7, CD103, CD25, CD36, CD11b, CD16, CD13, CD33, CD123, CD11c, CD14, CD64, CD15, cCD3, cMPO, cCD79a, and mLambda, which were identified as abnormal monoclonal B lymphocytes (Fig. 2). Based on the flow cytometry detections, two groups of abnormal cells existed in all the nucleated cell, of which CD19+ cells identified as abnormal monoclonal B lymphocytes (CD5 + CD10-) accounted for $58.10 \%$ of all the cells. The phenotype of the cells was also in consistence with B-CLL (score 5). In addition, 14.22\% abnormal myeloid primitive cells can be observed, which needed further examinations for verification.

As shown in Fig. 3A, FISH detections indicated that no rearrangement was detected in TP53/CEP17 (17P13.1/17p11.1-q11.1), RB1 (13q14), ATM (11q22.3), 12 (12p11.1-q11.1), and MYB (6q23) genes. However, regarding MLL (11q23) gene, the data showed that three signals were detected (one red and two green) and the positive rate was $39.5 \%$. MLL gene was labeled with red signal at 3 ' end and green signal at $5^{\prime}$ end. The fusion of genes will result in two yellow signals. The detection of one red and two green signals $\left(\left(5^{\prime} M L L \times 2,3^{\prime} M L L \times 1\right)[79 / 200]\right)$ represented the occurrence of gene rearrangement in $M L L$ gene ((KMT2A) (Fig. 3A).

Molecular detection using RT-qPCR showed that of all the 54 fusion genes related to leukemia, only KMT2A-ELL fusion gene t (11:10) (q23:p13.1) was detected (Fig. 3B). Further detections also showed that the sample were negative for $B$ lymphocyte immunoglobulin heavy chain (IGH) gene rearrangement and T lymphocyte receptor (TCR $\beta / T C R Y)$ gene rearrangement were.

High-throughput sequencing with the patient's bone marrow sample detected the expression status of all exon regions of 193 genes closely related to hematological tumors, including single nucleotide variation (SNV) and small insertion/deletion (INDEL). The results showed a missense mutation of p.V157F in the coding sequence of TP53 gene, and a frameshift mutation of p.V220fs and p.A382fs in the coding sequence of WT1. 


\section{Discussion And Conclusions}

The current study reported a rare occurrence of AML in a patient with untreated CLL. The patient declared intermittent fatigue, edema of both lower limbs, dyspnea, and occasionally fever up to $39^{\circ} \mathrm{C}$ during the last two months and the detecting results of CT showed that the patient had infections in bronchitis and both lungs. Thus, a comprehensive diagnosis was required immediately in this case. We then performed bone marrow morphology, bone marrow biopsy, bone marrow immunochemistry, karyotype analysis, flow cytometry, RT-qPCR, and high throughput sequencing with patients, which confirmed the co-occurrence of $C L L$ and $A M L$ in the patient.

The co-occurrence of CLL and AML in the same patient has been described in the last several decades (5-7). However, the mechanism driving the initiation of the symptom is still unclear. Some studies have hypothesized that the loss or deregulation of B-cell function led to the decreased immune surveillance, which subsequently induced the emergence of a new neoplasm. While other studies had suggested that the use of alkylating agents (a common treatment protocol for CLL) might increase the incidence of a second neoplasm. Regarding our case, no treatment for CLL had been given. The co-development of AML and CLL in the same patient might be attributed the alterations in the genetic background.

According to the RT-qPCR detection of fusion genes related to leukemia, only KMT2A-ELL was positive. The previous study reports that among all known KMT2A translocation partners, KMT2A-ELL fusions are present in $12 \%$ of adult AML, $7 \%$ of pediatric AML, and $15 \%$ of infant AML (9). However, the exact role of KMT2A-ELL in AML pathogenesis is still unclear, only with animal studies showing that the fusion protein provided an enhancing effect on the proliferative potential of hematopoietic progenitors (10). In addition to the KMT2A-ELL fusion, the results of high-throughput sequencing also showed that the patient was characterized by the mutations in TP53 and WT1 genes. It was demonstrated the coding sequence of TP53 gene had p.V157F missense mutation, which is a potential pathogenic mutation according to the analysis of ClinVar database (https://www.ncbi.nlm.nih.gov/clinvar/). Moreover, the COSMIC cancer database (https://cancer.sanger.ac.uk/cosmic) showed that this mutation was commonly detected in hematological tumors. TP53 is a classical tumor suppressor and is involved in various biological processes such as maintaining genome stability, regulating cell senescence, apoptosis, metabolism, and DNA repair. Mutations of TP53 are closely related to the initiation of AML, MDS, MPN, ALL, CLL, MM, which always indicates poor prognosis. Regarding WT gene, p. V220fs and p. A382fs frameshift mutations were found in the coding sequence of WT1 gene, which changed the translation reading frame and resulted in alterations in the amino acid sequence. The COSMIC cancer database shows that $p$. A382fs mutations are always detected in hematological tumors. WT1 protein is a transcription factor that plays an important role in the growth and differentiation of cells. Generally, mutations in WT1 gene are nonsense mutations, frameshift mutations, and splicing mutations, which are mainly found in AML, ALL, and also in MDS.

Collectively, we have reported a case in which there was simultaneous presentation of AML and CLL, and both forms of leukemia were demonstrated by morphology, cytometric analysis, and molecular studies. 
Moreover, the case in the current report showed fusion mutation of KMT2A-ELL gene, which might form the genetic basis of the occurrence of CLL and AML in the same patients. Our report provided value information for underlying the mechanism driving the co-occurrence of CLL and $A M L$, which will promote the treatment of the disease in clinic.

\section{Declarations}

\section{Funding information}

Not applicable

\section{Conflict of interest}

The authors disclose no conflict of interests

\section{Availability of data and material}

The data used to organize the MS has been provided along with MS.

\section{Ethics approval}

The study was approved by the ethic committee of The First Affiliated Hospital of China Medical University for related screening, inspection, and data collection.

\section{Consent to participate}

All the subjects had signed a written informed consent form, and all the procedures were performed in accordance with the Declaration of Helsinki.

\section{Consent for publication}

All the subjects had signed a written informed consent form, and all the procedures were performed in accordance with the Declaration of Helsinki.

\section{Acknowledgement}

Not applicable

\section{Authors' contributions}

Conceptualization: Shilin Zhang

Methodology: Sennan Qiao, Wei Han

Formal analysis and investigation: Zhonghua Du, Jing Bai, Fuming Zhang Writing - original draft preparation: Shilin Zhang 
Writing - review and editing: Sujun Gao

\section{References}

1. Travis LB, Curtis RE, Hankey BF, Fraumeni JF. Second Cancers in patients With Chronic Lymphocytic Leukemia. J Natl Cancer I. 1992;84(18):1422-7.

2. Zarrabi MH, Grünwald HW, Rosner F. Chronic lymphocytic leukemia terminating in acute leukemia. Arch Intern Med. 1977;137(8):1059-4.

3. Morita K, Mizuno T, Tanaka I, Suzuki H, Tanaka I, Miwa H, et al. [Acute myelogenous leukemia (M4) occurring during chronic lymphocytic leukemia]. Rinshō ketsueki. 1997;38(11):1212.

4. Tambaro FP, Garcia-Manero G, O"Brien SM, Faderl SH, Ferrajoli A, Burger JA, et al. Outcomes for patients with chronic lymphocytic leukemia and acute leukemia or myelodysplastic syndrome. Leukemia. 2016.

5. O'Neill BJ, McCredie KB, Raik E, Tauro GP. Mixed leukaemia: a report of three cases. Med J Australia. 1970;2(13):586-91.

6. Manoharan A, Catovsky D, Clein P, Traub NE, Galton DAG. Simultaneous or spontaneous occurrence of lympho- and myeloproliferative disorders: a report of four cases. Brit J Haematol. 2010;48(1):1116.

7. Raymond L, Arber DA, Brynes RK, Olivia C, Chang KL. Untreated chronic lymphocytic leukemia concurrent with or followed by acute myelogenous leukemia or myelodysplastic syndrome. A report of five cases and review of the literature. Am J Clin Pathol. 1999(3):373.

8. Ito S, Fujiwara SI, Mashima K, Umino K, Minakata D, Nakano H, et al. Development of acute myeloid leukemia in patients with untreated chronic lymphocytic leukemia. Ann Hematol. 2017;96(5):719-24.

9. Meyer C, Burmeister T, Gröger D, Tsaur G, Fechina L, Renneville A, et al. The MLL recombinome of acute leukemias in 2017. Leukemia. 2018;32(2):273-84.

10. Dimartino JF, Miller T, Ayton PM, Landewe T, Hess JL, Cleary ML, et al. A carboxy-terminal domain of ELL is required and sufficient for immortalization of myeloid progenitors by MLL-ELL. Blood. 2000;96(12):3887-93.

\section{Figures}



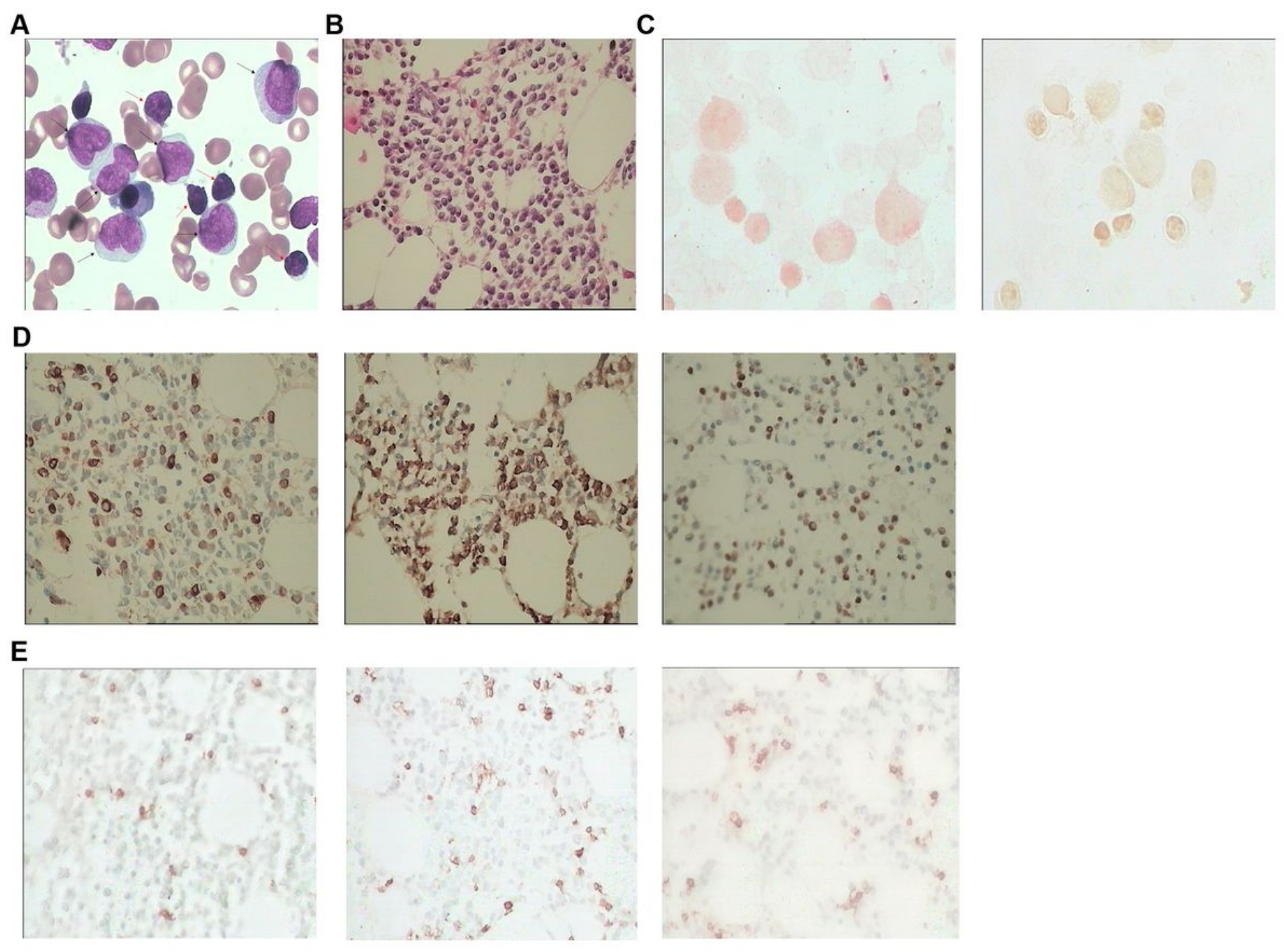

\section{Figure 1}

Histological detections of bone marrow samples. A, representative images of Wright staining. Black arrow, primary monocytes. Red arrow, mature lymphocytes. B, representative images of H\&E staining. C, representative images of stainings for ASD and a-NAE. D, representative images of IHC detection of CD34+ (left), CD117+ (middle), MPO+ (right). E, representative images of CD3 (left), CD20 (middle), CD5 (right). 

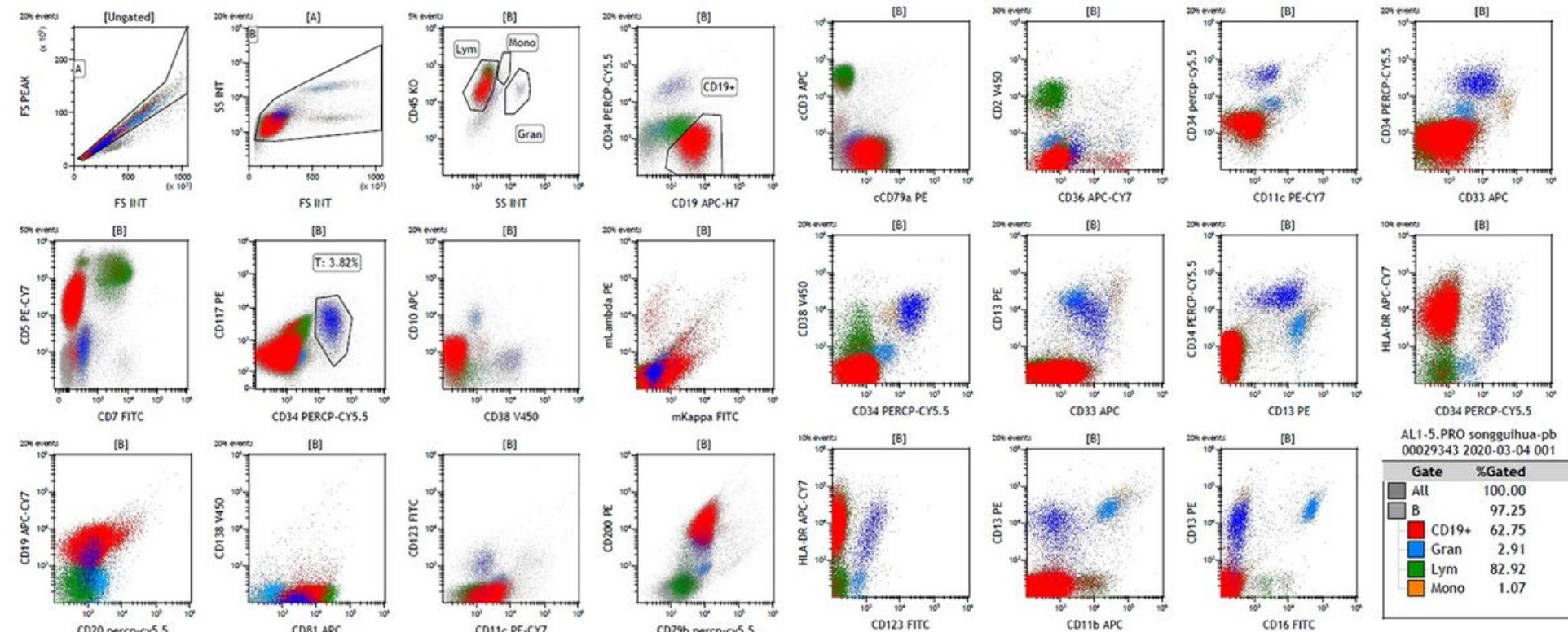

AL1.5.PRO songguihua -pb 000293432020.03 .04001 Gate \%Gated $\square$ All $\quad 100.00$ $\begin{array}{ll} & \\ \text { B } & 97.25\end{array}$ CD19+ 62.75 $\begin{array}{lr}\text { Gran } & 2.91 \\ \text { Lym } & 82.92\end{array}$ \begin{tabular}{lr}
\hline Lym & 82.92 \\
\hline Mono & 1.07
\end{tabular}
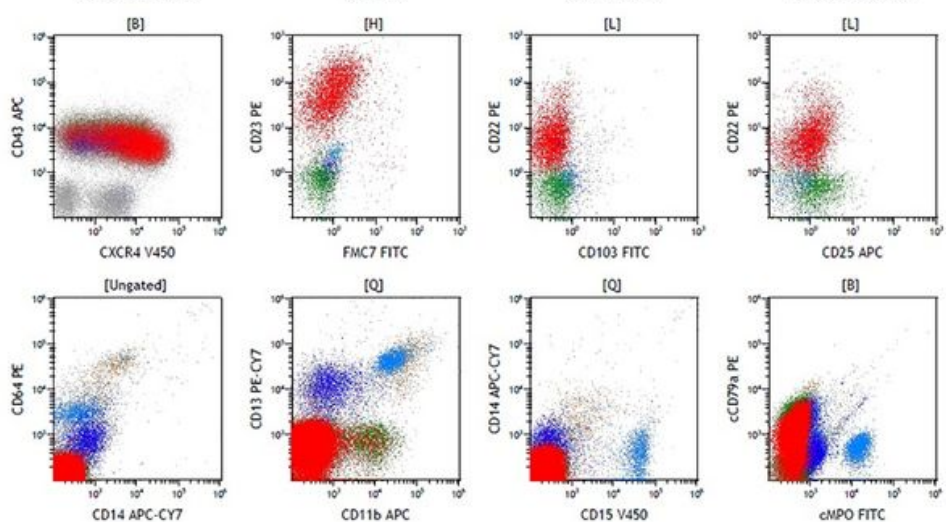

Figure 2

Representative images of flow cytometry detection of nuclear cells. 
A

TP53/CEP17

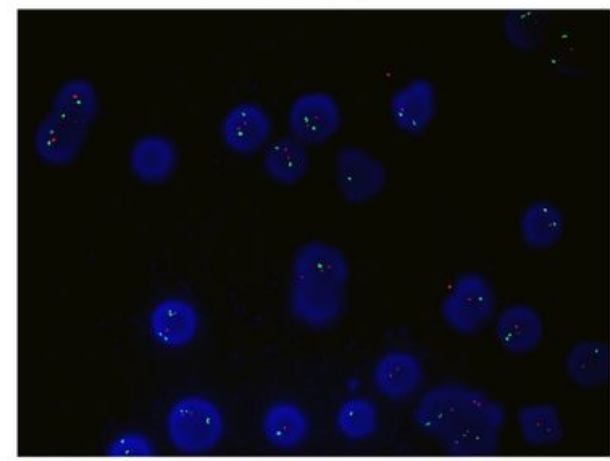

CEP12

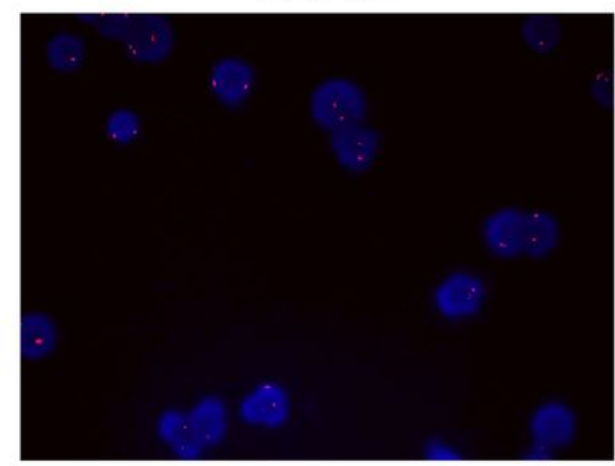

B

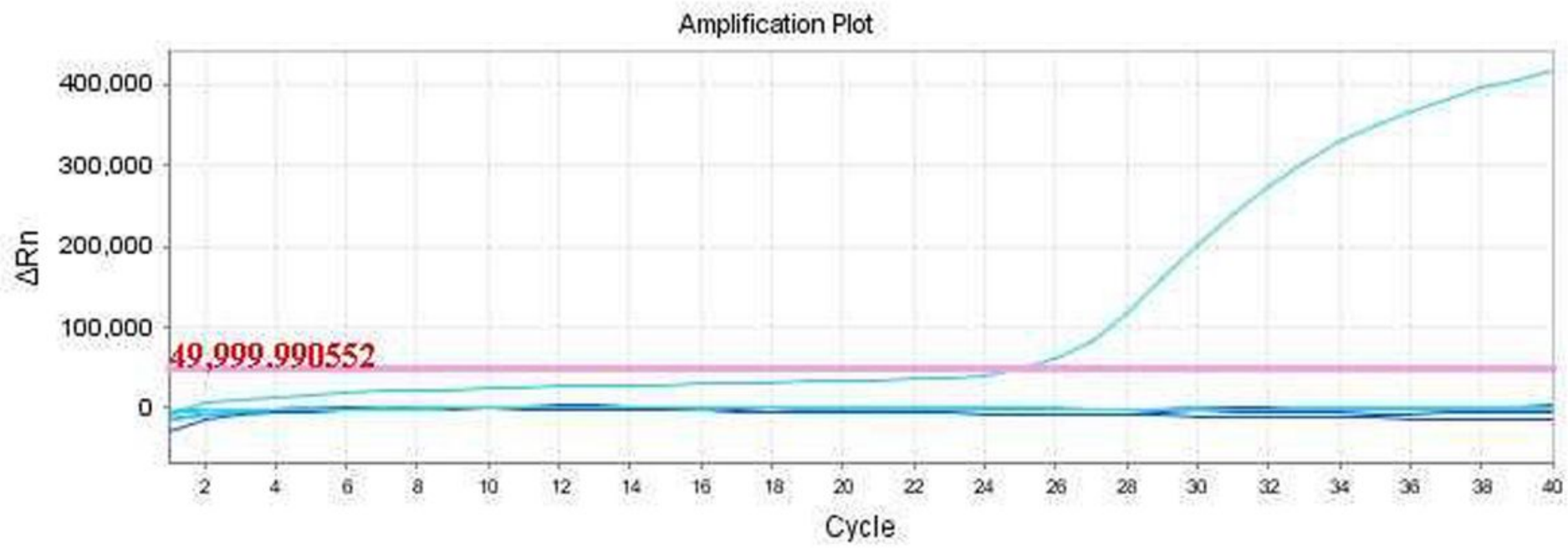

\section{Figure 3}

Representative images of FISH detections of gene mutation and amplification curve for KMT2A-ELL fusion gene. A, MLL gene was labeled with red signal at 3 ' end and green signal at 5 ' end. The detection of one red and two green signals represented the occurrence of gene rearrangement in MLL gene. $B$, amplification curve for KMT2A-ELL fusion gene. 